\title{
High Efficiency Photo-Induced Dissociation of Precursor Ions in a Tandem Time-of-Flight Mass Spectrometer
}

\author{
M. A. Seeterlin, P. R. Vlasak, D. J. Beussman, R. D. McLane, \\ and C. G. Enke \\ Department of Chemistry, Michigan State University, East Lansing, Michigan, USA
}

\begin{abstract}
High efficiency photo-induced dissociation (PID) has been demonstrated in a tandem timeof-flight mass spectrometer. This instrument focuses isomass ion packets to temporal and spatial dimensions similar to those of the focused laser pulses from a high power excimer laser. This high density overlap of photons and ions yields highly efficient fragmentation and also provides high resulution selection of specific precursor ion mass-to-charge ratio values. Using $193 \mathrm{~nm}$ photon excitation of the molecular ion of bromobenzene $(\mathrm{m} / z=156)$, fragmentation, collection, and PID efficiencies of $79 \%, 132 \%$, and $104 \%$, respectively, were obtained. Characteristic fragmentations of toluene, nitrobenzene, acetophenone, triethylamine, $N, N$-diethylformamide, $N$-methylacetamide, and cyclohexene have also been demonstrated. (J Am Soc Mass Spectrom 1993, 4, 751-754)
\end{abstract}

$\mathrm{T}$ HE tandem time-of-flight (TOF) instrument constructed in our laboratory has been designed to obtain complete tandem mass spectra from compounds eluting from a capillary gas chromatographic column while maintaining unit mass resolution for both precursor ion selection and product ion analysis. To meet this goal, the instrument needs to acquire at least 10 product spectra per second. Such spectral generation rates require maximum utilization of sample molecules and ions, efficient fragmentation of precursor ions, and array detection of the product ions produced. The use of continuous electron impact ionization with ion storage between pulsed extractions, TOF separation and reflectron focusing of precursor ions, pulsed laser-induced fragmentation, TOF separation and reflectron focusing of product ions, and array detection of all products formed meets each of these criteria.

Photo-induced dissociation (PID) as a means of inducing the fragmentation of ions has been used with several types of mass spectrometers. McGilvery and Morrison [1] and Krailler and Russell [2, 3] have directed the laser beam perpendicularly to the low density ion beam in the interaction region of, respectively, quadrupole and sector mass spectrometers. The low sensitivity with orthogonal excitation they observed was improved by the use of a coaxial geometry which placed a greater number of ions in the laser beam. Time-of-flight mass spectrometry in conjunction with

\footnotetext{
*Present address: The Proctor and Gamble Company, Sharon Woods Technical Center, 11450 Grooms Road, Cincinnati, OH 45241.

Address reprint requests to: Dr. C. G. Enke, Department of Chemistry. Michigan State University, East Lansing, MI 48824
}

PID has been implemented by several groups. Tecklenburg and Russell [4] have used a hybrid EB-TOF mass spectrometer with deceleration and PID occurring between the magnetic sector and the TOF mass spectrometer. Colby et al. [5] have performed TOF tandem mass spectrometry (MS/MS) analysis on benzene and aniline using photo-induced dissociation. These latter studies relied on the ability to selectively dissociate a chosen ion over all others in the source of a TOF mass spectrometer. Duncan and co-workers [6] have demonstrated the utility of PID in a reflectron TOF mass spectrometer by exciting gate-selected ions in the turn-around region of the reflectron. Schlag and coworkers [7] have demonstrated MS/MS by using linear TOF of gate-selected precursor ions, PID, and sequential focusing of the product ions with a reflectron voltage scanning technique.

Photo-induced dissociation is uniquely suited to the fragmentation needs of our tandem TOF mass spectrometer for several reasons. With this instrument, isomass ion packets are focused to temporal and spatial dimensions that are very similar to those of our focused laser pulse. The resulting high degree of spatial and temporal overlap provides optimal PID efficiency and permits high resolution selection of the precursor ion packet of interest. In addition, as opposed to collision-induced dissociation (CID) and surface-induced dissociation, the product ions formed by PID do not have the associated additional kinetic energy distribution acquired from a collisional process. Here we present a preliminary report of the achievement of high efficiency PID consistent with the overall instrumentation goals. 


\section{Instrument Description}

To maximize the PID fragmentation efficiency, an MS/MS instrument must expose the maximum number of ions produced in the source to as dense a population of phulons as possible. In addition, as many of the product ions formed must be detected as possible. The tandem TOF instrument constructed in our laboratory (Figure 1) provides this high overlap of ions and photons and efficient product detection. The ion source for the tandem TOF mass spectrometer utilizes continuous electron ionization with ion storage between pulsed extractions. The sample is continuously introduced via a heated capillary interface. Two opposing filaments and electron focusing elements form a dense electron cloud between the backplate and a grid, creating a potential well in which ions formed over the period between pulsed extractions are stored [8]. Ion extraction is accomplished by applying a high voltage pulse $(200 \mathrm{~V})$ to the backplate of the source followed by an additional acceleration of $550 \mathrm{~V}$. The high extraction field creates a space-focus plane at which isomass ions are focused to peaks with narrow temporal and spatial widths. These isomass packets separate in time due to their mass-to-charge ratio-dependent velocities and are refocused by reflectron 1 onto the interaction region. By setting the appropriate delay between the source extraction pulse and the laser triggering, precursor ions of any mass-to-change ratio can be selectively excited by the laser pulse at the interaction region. After PID, the product ions are postaccelerated to impart mass-to-charge ratio-dependent velocities. These product ions are separated in time and energy focused by reflectron 2 onto the detector. Because TOF mass spectrometry allows very high ion transmission efficiency and time-array detection is utilized, most of the product ions formed will be detected.

For precursor ion selection we rely on the laser pulse interacting with exactly one precursor mass-tocharge ratio value. The laser pulse is focused to a width of approximately $1 \mathrm{~mm}$. The isomass ion packets are also focused to $1 \mathrm{~mm}$ in length (resolution =
1200 FWHM). At $m / z$ 1000, the trailing edge (FWHM) of the $m / z 1000$ ion is separated from the leading edge of the $m / z 1001$ ion by $0.2 \mathrm{~mm}$. During the $17 \mathrm{~ns}$ temporal length of the laser pulse, the $m / z 1000$ ion packet travels $0.2 \mathrm{~mm}$. At higher mass-to-charge ratio values, the distance between ion packets becomes smaller than the distance traveled in $17 \mathrm{~ns}$, and unit resolution for precursor selection is not achieved. If greater resolution were required, focusing the laser beam to less than $1 \mathrm{~mm}$ or improvements in the precursor resolution (above 1200) could be made.

The laser used in this system is a Questek $2580 \mathrm{v} \beta$ excimer (Lambda-Physik, Acton. MA). Operating with ArF (193 nm) at $30 \mathrm{~Hz}$, this laser was measured to yield between 4 and $5 \mathrm{~W}$, or approximately $150 \mathrm{~mJ}$ per pulse. When focused by a cylindrical plano-convex lens down to the dimensions of the ion packet (1 $\mathrm{cm} \times 1 \mathrm{~mm}$ ), the laser pulse photon density is $1.46 \times$ $10^{18}$ photons $/ \mathrm{cm}^{2}$. Given that PID cross-sections have been determined to be between $10^{-17}$ and $10^{-19} \mathrm{~cm}^{2}$ [9] for ions such as $\mathrm{N}_{2} \mathrm{O}^{+}, \mathrm{CH}_{4}^{+}$, trimethylbenzene, butadiene, and hexatriene, the PID efficiency should theoretically be between $100 \%$ and $15 \%$ for these same molecules, and for molecules with similarly absorbing functional groups.

\section{Results and Discussion}

\section{Precursor Selection}

Figure $2 a$ is a spectrum of ions in the molecular ion region of bromobenzene. Ions in this spectrum have traveled through the entire instrument. With the laser on and the delay time between source extraction and laser firing set such that the laser pulse arrives at the interaction region at the same time as the precursor ions, interaction between the ions and photons occurs. Figure $2 \mathbf{b}-\mathbf{d}$ are the spectra obtained when the laser delay times are set at $86.40 \mu \mathrm{s}, 86.70 \mu \mathrm{s}$, and $86.97 \mu \mathrm{s}$ to coincide with the ion packet arrival times for massto charge ratio values 156,157 , and 158 , successively. The ion depletions associated with each of these times,

\section{Source}

Interaction Region

Reflectron 1

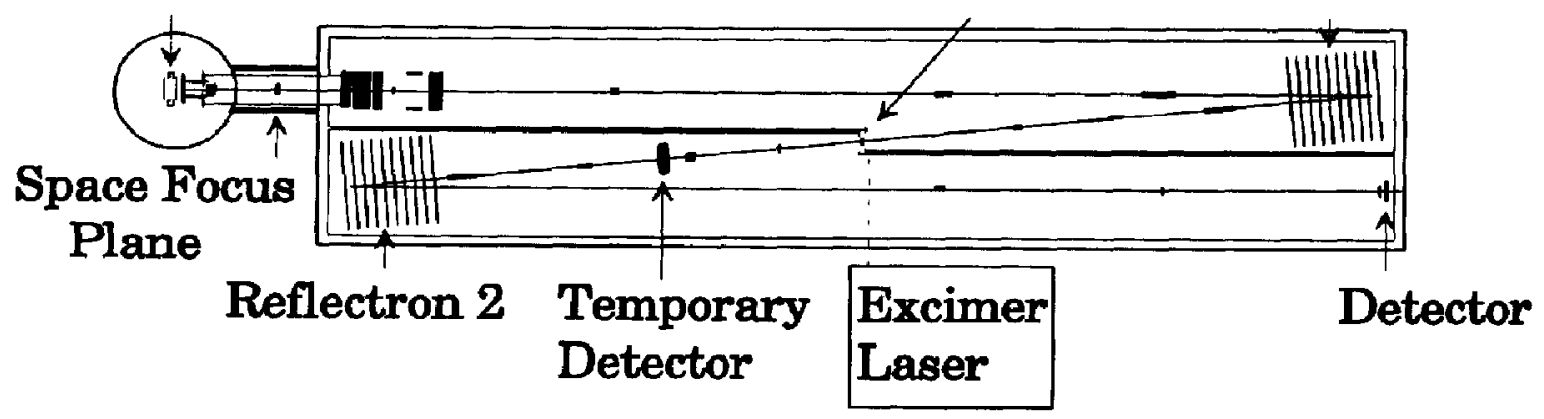

Figure 1. Diagram of the tandem time-of-flight mass spectrometer. 


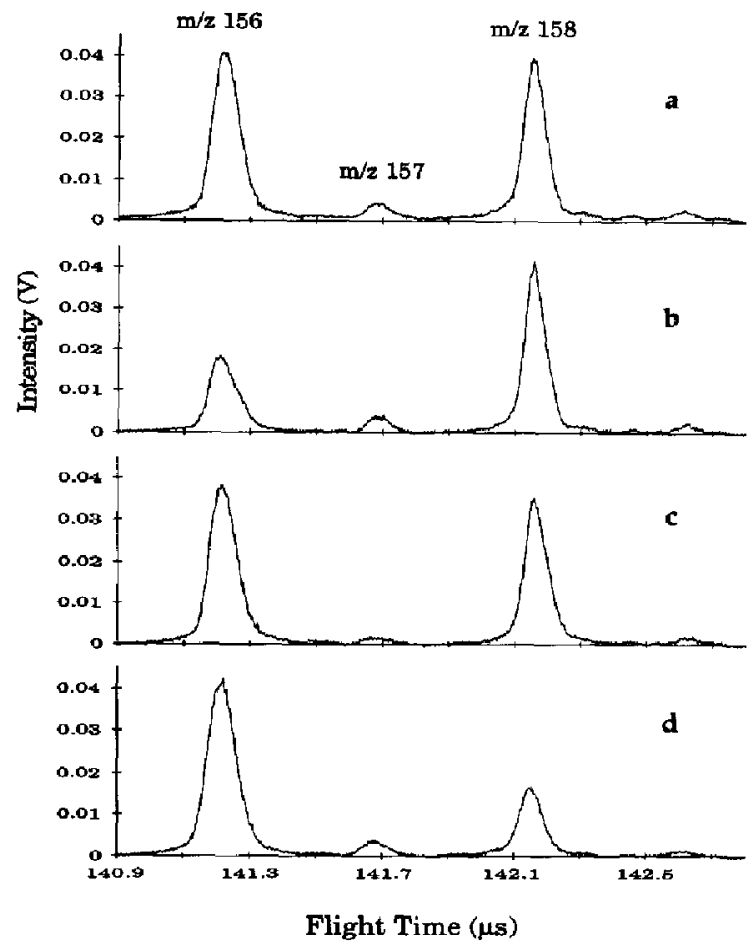

Figure 2. Spectra of the molecular ion region of bromobenzene (a) without the laser on, (b) with the laser delay set at $86.40 \mu \mathrm{s}$, (c) with the laser delay set at $86.70 \mu \mathrm{s}$, and (d) with the laser delay set at $86.97 \mu \mathrm{s}$. These spectra represent the sum of 200 transients.

being limited to the selected ion, demonstrate the effectiveness of precursor selection by laser timing adjustment. Calibration of delay times is easily accomplished owing to the linear dependence of flight time on the square root of mass-to-charge ratio. The ion arrival time jitter and laser thyratron discharge jitter were both measured to be less than 2 ns. Once the instrument is calibrated, selection of a new precursor ion simply involves changing the laser delay time to the calculated value for the desired precursor mass-tocharge ratio value. In addition, the day-to-day arrival time drift is less than 100 ns. Recalibration requires only three to four attempted laser delays. The bromobenzene example demonstrates that we are able to select only one precursor mass-to-charge ratio with the laser. Precursor ion areas have been depleted by $55.2 \%$, $56.3 \%$, and $51.8 \%$ for $m / z 156,157$, and 158 , respectively. This substantial depletion demonstrates that we are achieving considerable photon/ion interaction. To prove that the observed fragmentation is due to PID and not CID from laser-desorbed molecules, we have triggered the laser so that the laser pulse arrives at the interaction region $50 \mathrm{~ns}$ before the $\mathrm{m} / \mathrm{z} 156$ ion packet. No depletion was observed. In addition, the fact that we have selectively excited ion packets separated by 300 ns conclusively indicates that only PID is affecting the observed ion intensity.

\section{PID Efficiency}

The efficiency of the PID process is determined by the efficiency of fragmentation and the efficiency of collection of the ions. We can let $P$ and $P_{0}$ represent the precursor ion current with and without laser excitation, respectively, and $\Sigma F_{i}$ the total current of all fragment ions. The fragmentation efficiency is $L_{\mathrm{F}}-\Sigma \mathrm{F}_{\mathrm{i}} /\left(\mathrm{P}+\Sigma \mathrm{F}_{\mathrm{i}}\right)$, the collection efficiency is $\mathrm{E}_{\mathrm{C}}=\left(\mathrm{P}+\Sigma \mathrm{F}_{\mathrm{i}}\right) / \mathrm{P}_{\mathrm{o}}$, and the overall PID efficiency is the product $\mathrm{E}_{\mathrm{PID}}=\mathrm{E}_{\mathrm{F}} \times \mathrm{E}_{\mathrm{C}}=\Sigma \mathrm{F}_{\mathrm{i}} / \mathrm{P}_{\mathrm{o}}$ [10].

To perform these efficiency studies, a detector was placed approximately $20 \mathrm{~cm}$ beyond the interaction region, as depicted in Figure 1. This arrangement allows us to study the PID process independent of the effects of the second reflectron. When it is completely implemented, the second reflectron will improve product spectrum resolution. In addition, a low-voltage (10 V) repelling grid was placed immediately after the interaction region to deflect the multiphoton ionization products produced by the excitation of the background molecules present in the interaction region.

By using this arrangement the product spectrum for the molecular ion $(m / z 156)$ of bromobenzene was collected (Figure 3). In this case, the precursor ions have been separated by their mass-to-charge ratio-dependent velocities and focused at the detector. This product spectrum was obtained by subtracting the laser-off spectrum from the laser-on spectrum. Ion peaks at $\mathrm{t}=93.60 \mu \mathrm{s}, 93.39 \mu \mathrm{s}, 92.95 \mu \mathrm{s}, 92.50 \mu \mathrm{s}$, and $91.50 \mu$ s correspond to products ions with $6,4,3,2$, and 1 carbon atoms, respectively. The calculated fragmentation efficiency, collection efficiency, and overall PID efficiency are $56 \%, 116 \%$ and $65 \%$, respectively. The fragments observed in this product spectrum are similar to those observed in a normal $70 \mathrm{eV}$ electron ionization mass spectrum. Compared to the electron impact spectrum, the relative intensities of the lower mass-to-charge ratio fragment ions are enhanced in this PID product spectrum, indicating that either multiple single photon or multiphoton events are likely to be occurring.

Product spectra from precursor ions for bromobenzene, toluene, nitrobenzene, acetophenone, trieth-

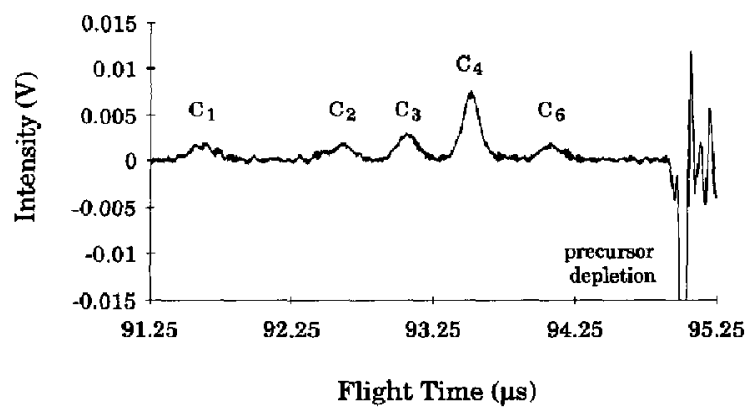

Figure 3. Product spectrum for the molecular ion $(\mathrm{m} / \mathrm{z} 156)$ of bromobenzene (sum of 200 transients). 
Table 1. PID results for several precursors

\begin{tabular}{|c|c|c|c|c|c|}
\hline Compound & $\begin{array}{l}\text { Precursor ion } \\
\qquad(m / z)\end{array}$ & $\begin{array}{c}\text { Laser pulse } \\
\text { energy } \\
\text { (mJ) }\end{array}$ & $\begin{array}{c}\text { Fragmentation } \\
\text { efficiency } \\
(\%)\end{array}$ & $\begin{array}{c}\text { Collection } \\
\text { efficiency } \\
(\%)\end{array}$ & $\begin{array}{c}\text { PID } \\
\text { efficiency } \\
(\%)\end{array}$ \\
\hline \multirow[t]{3}{*}{ Bromobenzene } & $\begin{array}{r}156 \\
77\end{array}$ & $\begin{array}{l}150 \\
160\end{array}$ & $\begin{array}{l}56 \\
30\end{array}$ & $\begin{array}{r}116 \\
93\end{array}$ & $\begin{array}{l}65 \\
28\end{array}$ \\
\hline & $156^{*}$ & 130 & 79 & 132 & 104 \\
\hline & $77^{*}$ & 130 & 44 & 113 & 50 \\
\hline Toluenc & 91 & 110 & 39 & 103 & 40 \\
\hline Nitrobenzene & 123 & 140 & 66 & 137 & 91 \\
\hline Acetophenone & 105 & 140 & 54 & 107 & 58 \\
\hline Triethylamine & 86 & 110 & 27 & 119 & 32 \\
\hline $\mathrm{N}, \mathrm{N}$-diethylformamide & 86 & 100 & 24 & 99 & 24 \\
\hline $\mathrm{N}$-methylacetamide & 73 & 120 & 29 & 94 & 27 \\
\hline Cyclahxene & 67 & 140 & 12 & 91 & 11 \\
\hline
\end{tabular}

* Precursor ians were focused at the interaction region instead of at the detector.

ylamine, $N, N$-diethylformamide, $N$-methylacetamide, and cyclohexene were collected. The compounds and precursor ions chosen demonstrate PID fragmentation of odd-electron, even-electron, aromatic, and nonaromatic species. Introduction of compounds of lower volatility awaits the implementation of a direct probe for sample introduction. Efficiencies are given in Table 1 . In most of these studies the ions were spacefocused at the detector rather than at the interaction region to improve product ion resolution. This resulted in reduced PID efficiencies. When reflectron 2 is used in the final instrument configuration to focus the product ions, space focus will be achieved at both the interaction region and at the detector. To determine the effect of improved focusing on PID efficiency ${ }_{r}$ two precursor ions (indicated by asterisks in Table 1) were studied with the space focus at the interaction region. As hypothesized, PID efficiencies were increased. Collection efficiencies of greater than $100 \%$ were sometimes observed. This is believed to be the result of greater detector gain for ions with higher velocity (lower mass-to-charge ratio) [11].

The overall PID efficiency of $104 \%$ achieved for bromobenzene is much higher than the $15 \%$ achieved by McIver and co-workers [12] in an ion cyclotron resonance instrument. Indeed, it is comparable to the high efficiencies achievable in the CID process in a triple quadrupole instrument (65\% for $n$-hexane) [10]. These results are evidence that PID can be an analytically useful fragmentation techuicue when implemented in a system with high ion/photon overlap.

\section{Acknowledgments}

We gratefully acknowledge the National Institutes of Health for supporting this work (NIH GM 44077). We also thank Prof. $H$. Wollnik for sharing his TOF technologies, Dr. R. Grix, Dr. G. Yefchak, and B. Gardner for help with optimizing TOF mass spectrometer performance, and $M$. Rabb for designing the electronics.

\section{References}

1. MoGilvery, D. C.; Morrison, J. D. Int. J. Mass Spectrom. Ion Physics 1978, 28, 81-92.

2. Krailler, R. E; Russell, D. H. Int. J. Mass Spectrom. Ion Processes 1985, 66, 339-351.

3. Krailler, R. E.; Russell, D. H. Anal Chem. 1985, 57, 1211-1216.

4. Tecklenburg, R. E. Ir.; Russell, D. H. Presented at the Pittsburgh Cunference; Atlinta, GA, March 1989; $p 488$.

5. Colby, S. M.; Yang, M.; Reilly, J. P. Presented at the Pittsburgh Conference; Atlanta, GA, March 1989; p 482.

6. LaiHing, K.; Cheng, P. Y.; Taylor, T. G.; Willey, K. F.; Peschke, M.; Duncan, M. A. Anal. Chem. 1989, 61, 1458-1460.

7. Weinkauf, R.; Walter, K.; Weickhardt, C.; Boesl, U.; Schlag, E. W. Z. Naturforsch 1989, 44a, 1219-1225.

8. Yefchak, G. E.; Puzycki, M. A.; Allison, J.; Enke, C. G.; Grix, R.; Holland, J, F,; Li, G.; Wang, Y.; Wollnik, H. Proceedings of the 38th ASMS Conference on Mass Spectrometry and Allied Topics; Tucson, AZ, Jume 1990; 540.

9. Dunbar, R. C. In: Gas Phase Ion Chemistry, Vol. 2. Bowers, M. T., Ed. Academic: New York, 1979; p 181.

10. Yost, R, A.; Enke, C. G.; McCilvery, D. C.; Smith, D.; Morrison, J. D. Int. J. Mass Spectrom. Ion Physics 1979, 30, 127-136.

11. Meier, R, Eberhardt, P. Int. J. Mass Spectrom. Ion Processes 1993, 123, 19-27.

12. Bowers, W. D.; Delbert, S.-S.; Mclver, R. T. Jr. Anal. Chem. 1986, $58,969-972$. 